\title{
EDIFICATE
}

I Congreso de Escuelas de Edificación y Arquitectura Técnica de España València, 4 y 5 de noviembre de 2021

Escuela Técnica Superior de Ingeniería de Edificación

UniversitatPolitècnica de València

Doi:https://doi.org/10.4995/EDIFICATE2021.2021.13396

\section{EI PFG como herramienta para la especialización en análisis integral y difusión del Patrimonio Arquitectónico}

\section{The PFG as a tool for specialization in comprehensive analysis and dissemination of Architectural Heritage}

\author{
Pedro Enrique Collado Espejo \\ ETS de Arquitectura y Edificación. Universidad Politécnica de Cartagena, pedroe.collado@upct.es
}

\begin{abstract}
The methodology developed in the ETS of Architecture and Building (ETSAE) of the Polytechnic University of Cartagena (UPCT), for the realization of Final Degree Projects (PFG) in the current Degree in Building Engineering is exposed, focused on architectural heritage. With the completion of the PFG the student shows how he has assimilated and integrated the training content received but it should also be an opportunity to enhance the specific and transversal competences of the degree. The text exposes the methodology, based on the motivation and involvement of the student, to develop the comprehensive analysis of the historic building and the dissemination of the PFG. The results obtained, after almost twenty years of experience and more than eighty PFG directed and focused on research, historical-constructive analysis and pathologies and the intervention proposal, for their future restoration and conservation, of historical buildings; show an effective methodology for the technical and human training of the student, especially in the field of Architectural Heritage. A successful experience if the awards that graduates have received in recognition of the quality of their PFG are taken into account.
\end{abstract}

Keywords: Final Degree Project, UPCT, Architectural Heritage, ETSAE, involvement, research, PFG methodology.

\section{Resumen}

Se expone la metodología desarrollada en la ETS de Arquitectura y Edificación (ETSAE) de la Universidad Politécnica de Cartagena (UPCT) para la realización de Proyectos Fin de Grado (PFG) en el actual Grado en 
Ingeniería de Edificación, centrados en el patrimonio arquitectónico. Con la realización del PFG el alumno muestra cómo ha asimilado e integrado los contenidos formativos recibidos pero también debe ser una oportunidad para potenciar las competencias específicas y transversales del título. El texto expone la metodología, basada en la motivación e implicación del estudiante, para desarrollar el análisis integral del edificio histórico y la difusión del PFG. Los resultados obtenidos, después de casi veinte años de experiencia y más de ochenta PFG dirigidos y enfocados a la investigación, el análisis históricoconstructivo y de patologías y la propuesta de intervención, para su futura restauración y conservación, de construcciones históricas; muestran una metodología eficaz para la formación técnica y humana del estudiante, especialmente en el ámbito del Patrimonio Arquitectónico. Una experiencia exitosa si se tienen en cuenta los premios que han recibidos los egresados en reconocimiento a la calidad de sus PFG.

Palabras clave: Proyecto Final de Grado, UPCT, Patrimonio Arquitectónico, ETSAE, implicación, investigación, metodología de PFG 


\section{Introducción}

A partir del curso académico 2000-2001, la Universidad Politécnica de Cartagena (en adelante UPCT), comienza a impartir la titulación de Arquitectura Técnica (diplomatura de tres cursos), integrando estos estudios en la Escuela Universitaria de Ingeniería Técnica Civil. En paralelo, la Declaración de Bolonia (1999) establecía el año 2010 como fecha para la implantación del llamado Espacio Europeo de Educación Superior (EEES), por lo que la UPCT aprobó, para el curso 2008-2009, implantar el Grado en Arquitectura y transformar el título de Arquitectura Técnica en el actual Grado en Ingeniería de Edificación (que comenzó a impartirse en el curso 2009-2010), además de crear la Escuela Técnica Superior de Arquitectura y Edificación (ETSAE), donde se integran, desde entonces, estas dos titulaciones (junto con el Máster en Patrimonio Arquitectónico y el Máster en Ciencia y Tecnología de la Edificación en Arquitectura).

Como se sabe, la implantación del EEES ha supuesto una profunda transformación de la Universidad, que se ha visto especialmente reflejada en los planes de estudios al pasar de diplomaturas de tres cursos a grados de cuatro, como en el caso de la titulación de Arquitectura Técnica/Ingeniería de Edificación. El modelo formativo y educativo que desarrolla el EEES sustituye el antiguo concepto de enseñanza, donde las clases magistrales y el libro de apuntes y prácticas formaban la base metodológica y modelo educativo (tanto en la enseñanza media como en el ámbito universitario), por el concepto de aprendizaje o, como también se ha definido, autogestión del aprendizaje. Un nuevo modelo que apuesta por la idea de "aprender a aprender" en lugar de la mera acumulación de conocimientos. Donde se incide en la evaluación contínua, la mayor tutorización del alumno, el aprendizaje autónomo..., y donde el profesor se convierte en un guía para el alumno y éste debe asumir gran parte de la responsabilidad de su proceso formativo (Collado, 2011). Y en este sentido, la elección del tipo de Proyecto Fin de Grado (genérico o específico, obra nueva o restauración, análisis teórico, realización de ensayos...), y cómo se afronte su realización, por parte del alumno y del profesor-tutor, es un ejemplo claro de las posibilidades de éxito o fracaso de lo que se persigue con la "autogestión del aprendizaje". Además, esta reforma del modelo de formación técnica y humana del alumno incluye la evaluación por adquisición de competencias. Es decir, los planes de estudios de las diferentes tutulaciones y las guías docentes de las asignaturas, establecen las competencias que el alumno debe alcanzar y el sistema de evaluación de éstas, dividiendo las competencias en específicas (del título y/o de la asignaturta) y genéricas o transversales, diferenciando estas últimas en tres bloques: instrumentales, personales y sistémicas, con sus correspondientes objetivos.

Por tanto, y teniendo en cuenta que el Reglamento de Trabajos Fin de Estudios en la UPCT establece que éste debe plantearse como "una herramienta fundamental para consolidar la adquisición de competencias por parte de los estudiantes, siendo en algunos casos la única actividad formativa en la que se puede abordar determinadas competencias trnasversales recogidas en los planes de estudios"; además de considerarlo como "un trabajo individual orientado a la aplicación y evaluación de competencias asociadas al título", la metodología de planificación y desarrollo del Proyecto Fin de Grado (PFG) en el actual Grado en 
Ingeniería de Edificación debe dar respuesta a estas necesidades. Es decir, el PFG debe enfocarse como un trabajo que integra y desarrolla la mayoría de contenidos formativos (técnicos y humanísticos) de la titulación y las competencias profesionales (específicas) y genéricas que se adquieren con, en nuestro caso, el título de Graduado en Ingeniería de Edificación (Collado, 2020). Así, tanto el Proyecto Fin de Carrera (en la antigua diplomatura de Arquitectura Técnica), como especialmente el actual PFG en Ingeniería de Edificación (IdE), debe planificarse y desarrollarse con una metodología adaptada a la consolidación y potenciación del máximo de competencias propias de la titulación, así como con un sistema que permita controlar y evaluar la calidad del trabajo y la adquisición de estas competencias por parte del autor del PFG. Además, la realización de este trabajo, como ocurre con el programa de prácticas, debe perseguir que el estudiante tenga una experiencia profesional que le ayude en su futura inserción en el mercado laboral (Raposo y Zabalza, 2011).

Este texto pretende exponer cómo se afronta y qué metodología se sigue en la UPCT para la realización de los PFG específicos, desde la época de la titulación de Arquitectura Técnica hasta la actual IdE, centrados en el análisis integral y propuestas de intervención en edificios que, básicamente, forman parte del Patrimonio Arquitectónico de la Región de Murcia, así como la evolución de esta metodología, con su adaptación a los nuevos requisitos de calidad, y los resultados obtenidos después de casi 20 años de experiencia.

\section{Objetivos. EI PFG como herramienta de conocimiento}

En general, las titulaciones técnicas tienen un enfoque técnico-científico muy acentuado y cuando se plantea un proyecto de anáilisis integral y propuesta de intervención en un edificio histórico se suele acometer desde ese rigor técnico y científico. Sin embargo, cuando estudiamos construcciones que forman parte del Patrimonio Arquitectónico o están protegidos como Monumento, no sólo tenemos que enfocar el análisis sobre la arquitectura y los materiales y sistemas constructivos propios del inmueble; también debemos tener muy presentes aspectos como el carácter histórico, simbólico, social y cultural de estos elementos; además de analizar y valorar su correcta integración y relevancia en el entorno urbano, natural y/o paisajístico en el que se ubican. Así mismo, tenemos que ser conscientes de que en las últimas décadas, términos como restauración, rehabilitación y conservación, están muy presentes en el debate social y cultural sobre cómo recuperar, adecuar e integrar los edificios antiguos en los actuales modos de vida. Un debate, más sociocultural que técnico, que persigue que estos edificios sigan en uso y en unas condiciones de habitabilidad acordes al momento actual, pero que no puede obviar la relevancia, singularidad e identidad de estas construcciones ni los variados e importantes valores que atesoran; valores históricos, arquitectónicos y constructivos evidentes, pero también valores sociales, documentales, simbólicos, culturales...

Por tanto, la formación y especialización del estudiante en el campo del Patrimonio Arquitectónico debe enfocarse, como mínimo, en un doble sentido: el técnico y el humanístico. Y el PFG puede convertirse en una herramienta muy eficaz para que el 
alumno de IdE desarrolle la mayoría de los conocimientos generales adquiridos y, en el campo de la arquitectura monumental, la formación específica en tipologías constructivas, metodologías de análisis, criterios y técnicas de intervención, los conceptos de autenticidad, legibilidad y reversibilidad, etc. Todo ello sin olvidar que, con la realización del TFG, y en cumplimiento de la Guía Docente, debemos estimular y potenciar en el alumno competencias instrumentales como: capacidad de análisis y síntesis, capacidad de organización y planificación del trabajo, capacidad de gestionar la variada y compleja información que se va generando, el razonamiento crítico-constructivo, la comunicación oral y escrita (el PFG debe estar bien redactado), y la capacidad y madurez para la toma de decisiones. En cuanto a las competencias personales, se afianzarán: el aprendizaje autónomo, las habilidades en las relaciones interpersonales y la capacidad de trabajar en equipo, que practican al tener que analizar las últimas intervenciones realizadas en el edificio y comentar éstas con el arquitecto director y/o con algún técnico de la empresa que las llevó a cabo. Igualmente, el PFG debe favorecer el impulso de las competencias sistémicas (creatividad e innovación en las propuestas de intervención, iniciativa y motivación por la calidad del trabajo), que serán muy valoradas por el tribunal evaluador del PFG al constituir el resultado final de todo el trabajo teórico-práctico realizado.

\section{Metodología para el desarrollo del PFG específico en Patrimonio}

Hasta hace unos años, el "levantamiento arquitectónico", general y en detalle, se consideraba la herramienta básica, habitual, para el conocimiento arquitectónico, material y constructivo de los edificios; conforme lo vamos dibujando, reconocemos y comprendemos, material y constructivamente, el edificio. Por ello, la realización y definición de los planos (en CAD a partir de principios de los 90), tenía una carga de trabajo y una valoración muy importante en los PFG, limitándose el análisis histórico y descriptivo del edificio a una pequeña memoria justificativa. Con el desarrollo de la metodología de trabajo que vamos a exponer se pretende conseguir un "conocimiento integral", completo y en profundidad, del edificio/conjunto arquitectónico. Este análisis no sólo debe permitir el conocimiento de su materialidad física, sino que abarca todo lo que concierne a su historia arquitectónica, social y cultural, su identidad, sus valores y significados (que pueden haber cambiado a lo largo del tiempo). Un conocimiento integral básico para poder asumir, con las mayores garantías, la complejidad de cualquier intervención en el Patrimonio Arquitectónico.

Como punto de partida, tenemos que tener muy en cuenta que los PFG los realizan alumnos que están en el final de su formación académica universitaria (por lo que disponen de amplios conocimientos teóricos y prácticos de su titulación), que serán tutorizados por un profesor (con más o menos experiencia en esta labor), pero que dependiendo de las circunstancias personales del estudiante, en muchos casos compaginando la realización del PFG con trabajos (Pérez et al., 2019), y no siempre relacionados con sus estudios universitarios, afrontarán este nuevo reto de muy diversas formas. Por tanto, y en base a nuestra experiencia, nos atrevemos a afirmar que el profesor-tutor de un PFG específico centrado en el análisis integral de un edificio histórico debe tener presente que: 
- Se trata de un trabajo original, que debe ser evaluado por un Tribunal y reflejar los conocimientos, habilidades y competencias (específicas y genéricas), que el alumno debe haber adquirido a lo largo de su formación universitaria.

- EI PFG tiene una carga de 12 ECTS, no es una Tesis Doctoral, pero sí debería servir para que el alumno adquiera un modelo correcto de investigación y referenciación documental y desarrolle su capacidad observadora, reflexiva y crítica.

- El profesor-tutor debe facilitar la realización del PFG, no "controlarlo"; debe ayudar en la evolución y aprendizaje del alumno que debe ser el que busque soluciones a los retos que se plantean con el desarrollo del trabajo.

- El profesor-tutor debe comportarse como un técnico experimentado que sirve de guía y apoyo en todo lo necesario para el correcto aprendizaje del estudiante, haciendo que esta experiencia sea lo más enriquecedora posible (Zabalza, 2011)

- Cuando las propuestas del alumno o la orientación del trabajo no es el adecuado, el tutor debe saber "canalizar" el PFG pero sin desmotivar al estudiante, éste debe sentir que su opinión, su participación es fundamental.

- La relación entre tutor y alumno ha de ser fluida, de confianza y abierta a plantear propuestas creativas e innovadoras. Por ello, las tutorías presenciales u online deben ajustarse a la evolución del trabajo pero nunca renunciar a ellas. En una situación de pandemia, plataformas como TEAMS facilitan la interacción tutor-alumno.

- La implicación y responsabilidad del estudiante depende de él mismo pero también de la actitud del tutor. El profesor debe favorecer e incentivar la participación del estudiante (Sánchez et al., 2015), su razonamiento crítico y toma de decisiones y su autoevaluación. Las tutorías son fundamentales para canalizar la implicación del estudiante en este proceso de compromiso con el aprendizaje.

- Los PFG específicos favorecen la implicación del alumno, especialmente cuando es éste el que elige el tema y los objetivos del trabajo a realizar.

- El análisis integral de un edificio histórico aumenta la sensibilidad hacia la conservación y correcta valoración del Patrimonio. El trabajo es una poderosa herramienta, didáctica y divulgativa, al servicio del Patrimonio Cultural.

- Manejar mucha información no es sinónimo de entenderla. El exceso puede "bloquear" al alumno. Éste debe aprender a seleccionar y procesar la información.

- La originalidad del PFG será comprobado con un programa antiplagio (Turnitin en el caso de la UPCT), lo que implica gestionar y procesar correctamente la información (sobre todo histórica y legislativa) que se maneja.

- Los alumnos se implican cuando se sienten respetados y valorados, por ello, se les debe tratar como profesionales. 
- Los alumnos motivados son más receptivos y aprenden más. La motivación tiene una influencia importantísima en el aprendizaje (Sancho, 2016).

- Establecer una metodología de desarrollo del PFG ayuda a planificar y controlar el trabajo pero debe ser flexible y adaptarse a la situación personal del estudiante (dedicación, horarios de trabajo, situación familiar...) y a las características y objetivos del trabajo específico a desarrollar.

- El alumno de PFG suele compaginar éste con un trabajo profesionalpor lo que, en ocasiones, no dispone de tiempo para finalizar el PFG en el plazo establecido (Pérez et al., 2019), lo que suele conllevar desmotivación. El profesor-tutor debe ser comprensivo pero también debe insistir en motivar y comprometer al estudiante.

- Analizar la/s última/s intervención/es realizada/s en el edificio origen del PFG implica que el estudiante debe contactar con la dirección facultativa, constructora y promotor de las obras. Compartir conocimientos con profesionales es una experiencia muy enriquecedora y motivadora para el estudiante, además de potenciar su capacidad de análisis y síntesis y sus habilidades en las relaciones interpersonales.

Además de todo esto, realizar un PFG sobre un edificio histórico, trabajar en el Patrimonio Cultural, se convierte en un proceso de sensibilización y compromiso ético hacia todo lo que representa el Patrimonio. Por tanto, este trabajo debe plantearse también como una oportunidad para el desarrollo emocional y la formación integral del estudiante hacia el conocimiento y respeto al Patrimonio Cultural, con todo lo que ello conlleva socialmente. Asi mismo, este tipo de PFG exige un alto grado de dedicación, compromiso y conocimientos por parte del profesor-tutor (Alfaro et al., 2012); sólo así podrá orientar y ayudar al alumno.

\section{Desarrollo del PFG específico en Patrimonio. Resultados}

A partir de estas premisas, el PFG se divide básicamente en tres bloques: análisis histórico, constructivo, sociocultural y de las intervenciones realizadas; análisis del estado actual de conservación; y propuesta razonada de intervención para la correcta conservación y uso. Estos tres bloques deben organizarse teniendo en cuenta que la originalidad del trabajo será comprobada con el programa antiplagio y éste no analiza documentación gráfica (planimetría) y cuando se analiza el cumplimiento de la normativa actual en Patrimonio Cultural, accesibilidad, protección, mantenimiento, ahorro energético, etc., el porcentaje de "similitud" puede ser alto, pues hay que hacer referencia a la normativa en cuestión. Por ello, la ETSAE aprobó que los PFG desarrollados en esta Escuela deben entregarse en tres archivos pdf independientes. Uno con los análisis histórico-constructivos, estado de conservación y propuesta de intervención; otro con la documentación planimétrica; y un tercero con al análisis del cumplimiento de la normativa y los correspondientes anexos. Sólo el primer archivo (el de los análisis teóricos) se analizará con el programa antiplagio.

El primer paso para desarrollar el PFG es que el/la alumno/a elija el edificio histórico sobre el que quiere trabajar. Los profesores podemos ofertar trabajos/edificios pero si es elección del estudiante la motivación e implicación de éste casi está garantizada. La busqueda del 
edificio ya forma parte del PFG, potenciando competencias como la iniciativa y espíritu emprendedor, el aprendizaje autónomo, capacidad de análisis, toma de decisiones... El edificio debe poder ser inspeccionado sin grandes inconvenientes y la publicación del PFG, en abierto (en el repositorio de la UPCT), también debería estar garantizada (los inmuebles gestionados por algunas administraciones no siempre cumplen estas premisas y los propietarios particulares también pueden poner objeciones a visitar la totalidad del edificio).

Elegido el edificio, el trabajo comienza con la elaboración del índice general. Un documento "abierto", que se irá adecuando al desarrollo del PFG pero que sirve para organizar y planificar los contenidos a desarrollar. El primer capítulo se centrará en exponer los objetivos que se persiguen y la metodología empleada. Desde el primer momento, el alumno debe saber qué objetivos tiene que alcanzar. Normalmente, el análisis del estado de conservación, junto con la memoria histório-cultural, indicará la propuesta de intervención, que debe ser valorada económicamente (no con un extenso presupuesto pero sí con una valoración global de las intervenciones propuestas). Por tanto, el objetivo suele ser la intervención sobre las patologías detectadas y la correcta adecuación del edificio al uso actual o a otro uso (respetuoso con los valores patrimoniales del edificio y cumpliendo las diferentes normativas que le afecten). La búsqueda, selección y procesado de la documentación escrita e información oral (debidamente contrastada), debe enfocarse al conocimiento integral del inmueble y a los objetivos que se persiguen.

Normalmente, el trabajo empieza con un breve análisis de la historia del municipio en el que se ubica el edificio y un estudio más amplio de la época histórica y sociocultural en que se construyó. Continúa con el estudio histórico-cultural del propio edificio (quién lo proyectó y construyó, por qué, cómo ha evolucionado el inmueble), el análisis descriptivo (volumétrico y compositivo, posibles añadidos y/o desmontajes/demoliciones, elementos artísticos y decorativos) y el estudio constructivo (materiales y sistemas constructivos tradicionales y/o contemporáneos, su compatibilidad). Así mismo, se analizan las diferentes intervenciones que el edificio ha tenido durante su vida, poniendo especial énfasis en las últimas. Como se ha comentado, es fundamental que el alumno contacte con la dirección facultativa, la constructora y el promotor de esas intervenciones para documentarlas correctamente y hacer un análisis crítico-constructivo, que le servirá para su propuesta razonada de intervención integral. Toda esta información es básica para entender el valor Patrimonial del inmueble, su evolución e influirá en la toma de decisiones para su conservación.

Lógicamente, la realización de planos generales y de detalle ayudarán a entender el edificio, por lo que se realizará un completo levantamiento gráfico y de precisión (en los últimos años con escáner láser 3D y fotogrametría digital), con planos de alzados, plantas, secciones y detalles constructivos. Al tratarse de construcciones antiguas, hay que buscar el patrón de medida con el que se proyectó y construyó el edificio, sabiendo que hasta 1849 no se implanta en España el Sistema Métrico Decimal (uniformidad metrológica con el metro como medida básica). Por tanto, los planos deben reflejar la escala y el patrón de medida que se usó originalmente (en el caso del antiguo Reino de Murcia solía ser la vara de Burgos o vara castellana) y su correspondencia con el patrón actual. 
El estudio del estado de conservación, con el análisis de las patologías detectadas (localización e identificación de deterioros y lesiones, grado de incidencia/peligro, hipótesis sobre las causas y su evolución, necesidad o no de apuntalamientos u otras medidas urgentes, planimetría específica de patologías, fichas de deterioros/lesiones por elementos constructivos, etc.), es fundamental para afrontar posteriormente la propuesta de intervención. Así mismo, este análisis es una de las grandes aportaciones que el estudiante hace a favor de la restauración y conservación del edificio en cuestión, pues una copia del PFG se suele entregar al propietario del inmueble y otra al Servicio de Patrimonio Histórico, dependiente de la Direcc. Gral. de Bienes Culturales de la CARM, con lo que esto conlleva.

Y como paso previo a la propuesta de intervención, se realiza un estudio del grado de protección del edificio (según el PGMO y la normativa en Patrimonio Cultural en vigor) y las repercusiones respecto al estado actual de conservación, así como el cumplimiento de otras normativas, como la de accesibilidad, si es un edificio de pública concurrencia, la de eficiencia y ahorro energético, seguridad, etc.

Por tanto, este amplio conocimiento del edifico capacita al estudiante para plantear una intervención adecuada a las características y singularidad del inmueble, que resuelva las patologías detectadas y que sirva para adaptarlo a un uso que siempre debe ser respetuoso con todos sus valores patrimoniales. Así mismo, el PFG incluye una estimación económica, un presupuesto no excesivamente desglosado pero que sí permite estimar, por fases, las diferentes intervenciones que se han propuesto. $Y$ todo ello tutorizado por un profesor pero desarrollado por el/la alumno/a y bajo las premisas de conocimiento, implicación, responsabilidad y calidad del trabajo expuestas anteriormente.

Finalmente, el PFG incluye un capítulo de conclusiones en el que se hace un resumen del trabajo realizado, se comenta cómo se ha planificado y desarrollado éste, los objetivos que se marcaron y los logros conseguidos. Se destaca la importancia de los estudios realizados y las propuestas de intervención, razonadas y justificadas, para la conservación integral del edificio histórico analizado. Así mismo, la última reforma del Reglamento de Trabajos Fin de Estudios de la ETSAE obliga a entregar al menos dos láminas, impresas en formato A2, "que muestren el trabajo realizado". Este ejercicio de concreción del PFG desarrolla y potencia competencias instrumentales como la capacidad de análisis y síntesis, la gestión de la información documental y oral, la comunicación escrita y el razonamiento crítico, entre otras; competencias que serán muy importantes en el futuro profesional del estudiante.

Con la defensa pública del PFG, ante un Tribunal compuesto por tres profesores de la ETSAE, el alumno estimula su capacidad de exponer y defender sus ideas y trabajo, y lo debe hacer de manera clara, eficaz y motivadora, y en un tiempo máximo de 20 minutos. Este ejercicio potencia sobremanera la capacidad de síntesis y comunicación del alumno, fortaleciendo su lenguaje verbal (vocalización, volumen, fluidez, entonación...), no verbal (vestimenta apropiada, posición corporal, gesticulación, tensión visual con el tribunal y el auditorio...), y las competencias de creatividad e innovación, al tener que elaborar una presentación que reafirme y complemente su discurso, a la vez que lo diferencia y distingue positivamente del resto de PFG (de los ya defendidos y de los que, seguramente, se 
defienden también en la misma sesión). Toda una experiencia vital que le ayudará en su futuro profesional como arquitecto técnico, especialmente como dirección facultativa.

La publicación en formato pdf en el repositorio de la UPCT, en abierto, de los numerosos PFG desarrollados con esta metodología de conocimiento integral permite difundir tanto la propia metodología como los resultados, dando a conocer y poniendo en valor los edificios analizados. Además, las Jornadas de Patrimonio Cultural de la Región de Murcia, organizadas conjuntamente por la Direcc. Gral. de Bienes Culturales de la CARM y la UPCT y que se celebran anualmente (en 2021 será la XXVII edición), incluyen un bloque temático para la exposición oral y publicación, en el Libro de Actas, de los mejores PFG de la UPCT defendidos en el año anterior a las Jornadas y relacionados con el análisis integral del Patrimonio Arquitectónico, con lo que los egresados también disponen de un evento, donde participan numerosos profesionales que trabajan en el Patrimonio Cultural, para mostrar sus conocimientos y especialización. Un motivo más para potenciar la implicación y motivación del estudiante en la realización, con calidad, de su PFG

Por último, destacar que en 2017 el Colegio Oficial de Aparejadores, Arquitectos Técnicos e Ingenieros de Edificación de la Región de Murcia creó los "Premios COAATIEMU a Trabajos Final de Grado y de Máster", con el objetico de "dar visibilidad y divulgar el importante trabajo de investigación que se hace en las Universidades por parte de los arquitectos técnicos, reconociéndoles el gran esfuerzo y dedicación invertidos, y premiando a aquellos cuyos trabajos destaquen, por el interés que representan para la profesión o bien por el planteamiento del tema tratado y las conclusiones aportadas". A estos premios puede concurrir cualquier colegiado/a o precolegiado/a del COAATIEMU así como cualquier recien egresado/a de la UPCT, Universidad Católica San Antonio (UCAM) u otras universidades politécnicas con PFG relacionados con la edificación en la Región de Murcia. Y en las tres primeras ediciones, el $1^{\circ}$ Premio ha recaído en PFG realizados por alumnos de IdE de la UPCT y desarrollados con la metodología de trabajo expuesta en este texto. En 2017, el $1^{\circ}$ Premio recayó en el PFG con título "Arquitectura tradicional en Cehegín. El Hospital de la Real Piedad. Análisis histórico, constructivo y de patologías. Propuesta de intervención", realizado por el egresado D. Francisco Jesús Ondoño Llorente. En 2018, en el trabajo "Balneario Floridablanca en San Pedro del Pinatar. Análisis histórico-constructivo y de patologías. Propuesta de intervención", de D. Francisco Javier Tárraga Martínez que, además, obtuvo un reconocimiento a nivel nacional al ser galardonado con el $3^{\circ}$ Premio en la "Primera Edición de los Premios a Trabajos de Fin de Grado que concede el Consejo General de la Arquitectura Técnica de España". Y en 2019, en el PFG con título "Rehabilitación de la arquitectura histórica. Análisis integral de los trabajos de rehabilitación de pabellones del Cuartel de Artillería en Murcia", desarrollado por D. Víctor San Bernardo Hernández. Así mismo, otros trabajos desarrollados con esta metodología han recibido también el reconocimiento del COAATIEMU. En la edición de 2017, el PFG "Las murallas medievales de Mula (Murcia). Estudio integral y propuesta de restauración de la muralla del albacar", realizado por D. Juan Fernández Del Toro, obtuvo el $3^{\circ}$ Premio; y en la edición de 2018, el PFG con título "Edificios ferroviarios en el término municipal de Mula. Análisis 
histórico-constructivo y de patologías. Propuesta de intervención", de D. Francisco José Huertas Fernández, obtuvo el $2^{\circ}$ Premio. Por tanto, unos reconocimientos también a la calidad de estos PFG y a su importancia como herramienta para desarrollar y potenciar competencias genéricas y específicas del graduado en IdE y como recurso para la especialización de los técnicos en el campo del Patrimonio Arquitectónico.

\section{Algunas conclusiones}

Desde el curso 2000-2001 se imparte la titulación de Arquitectura Técnica en la UPCT, que se adaptará al EEES en el curso 2009-2010, impartiéndose como Grado en Ingeniería de Edificación. El modelo formativo y educativo que se desarrolla con los criterios del EEES incide en el concepto de autogestión del aprendizaje, potenciando la evaluación contínua y por adquisición de competencias (genéricas y específicas), y el aprendizaje autónomo, con lo que el estudiante adquiere mucho más protagonismo y responsabilidad en todo este proceso. Por tanto, el PFG debe realizarse con una metodología que dé respuesta a todas estas cuestiones. Es decir, el trabajo tiene que planificarse y desarrollarse con una metodología y unos criterios técnico-científicos y de carácter teórico-práctico (muy acentuados en las carreras técniccas), complementados con conceptos y planteamientos más "humanísticos" (especialmente cuando se analizan edificios monumentales). Unos trabajos adaptados a la consolidación y potenciación del máximo de competencias propias del egresado en IdE, así como con una metodología que permita controlar y evaluar la calidad del trabajo y la adquisición de esas competencias.

En el caso de PFG específicos y enfocados al análisis de edificios protegidos, incluso monumentales, la formación y especialización del estudiante en este campo y con la premisa del conocimiento integral, debe convertirse en uno de los grandes objetivos.

La motivación, implicación y responsabilidad del estudiante son básicas para la correcta realización de un PFG. Estas actitudes dependen, básicamente, del estudiante pero también pueden y deben favorecerse y potenciarse con el compromiso positivo, flexible y colaborador del profesor-tutor. La tutorías presenciales (u online, si no son posibles las presenciales) son muy importantes en este proceso de gestión del aprendizaje y motivación del estudiante, y en ellas el estudiante debe sentirse escuchado, respetado y valorado por su tutor. El alumno puede ser impulsivo y plantear propuestas no justificables, por lo que la labor del tutor debe centrarse en canalizar esta situación, pero nunca cohibir al alumno.

La metodología que se ha implantado y se sigue en la ETS de Arquitectura y Edificación de la UPCT, para desarrollar los PFG del Grado en Ingeniería de Edificación enfocados al análisis y propuesta de conservación de construcciones históricas, se basa en la idea de que estos Proyectos sirvan para fomentar y potenciar las competencias específicas y genéricas del egresado en IdE, así como en convertirse en una herramienta y recurso eficaz para la especialización en el ámbito del Patrimonio Arquitectónico y Cultural. Los casi veinte años de experiencia permiten afirmar que la realización de un riguroso análisis integral (estudio histórico-constructivo, planimétrico y de patologías detectadas), junto con las propuestas técnicas de intervención del edificio histórico para su futura restauración y 
conservación, se ha mostrado como una muy buena herramienta para la formación técnica y humana del estudiante. Una experiencia exitosa si se tienen en cuenta los premios que han recibidos los egresados de IdE de la ETSAE en reconocimiento al esfuerzo realizado, la calidad de la investigación, su difusión y el interés que representan estos PFG para el ejercicio de la profesión de arquitect técnico/ingeniero de edificación.

Por último, insistir en que el PFG debe organizarse y desarrollarse para mostrar cómo el estudiante ha asimilado e integrado los contenidos formativos recibidos durante su formación universitaria, pero también debe plantearse como una oportunidad para potenciar las competencias específicas y transversales del título en Arquitectura Técnica/Ingeniería de Edificación. Y en el caso de trabajos específicos en Patrimonio Arquitectónico, el PFG debe abordarse también como una oportunidad para el desarrollo emocional y la formación técnica integral del estudiante hacia el conocimiento, respeto y difusión del Patrimonio Cultural, con todo lo que esto conlleva social y profesionalmente.

\section{Referencias}

ALFARO GONZÁLEZ, J.; ARTEAGA MARTÍNEZ, J.J.; y VALVERDE CANTERO, D. (2012). Competencias básicas y herramientas docentes para la tutorización de Proyectos Fin de Grado en la titulación de Grado en Ingeniería de Edificación. En Las competencias básicas. Competencias profesionales del docente. Universidad de Castilla-La Mancha; pp. 761-770.

COLLADO ESPEJO, P.E. (2011). Autogestión del aprendizaje en el estudio del Patrimoniuo Arquitectónico: la experiencia del Taller de Restauración Monumental en la UPCT. En Congreso Internacional de Innovación Docente 2011. Cartagena. CRAI-Biblioteca. Universidad Politécnica de Cartagena; pp. 330-331 (abstract)

COLLADO ESPEJO, P.E. (2020). El Trabajo Fin de Grado: una oportunidad para la especialización en el análisis y difusión del Patrimoniuo Arquitectónico. En Anuario 3. Colección Docencia, Innovación, Investigación. Cartagena. CRAI-Biblioteca. Universidad Politécnica de Cartagena; pp. 130-135.

UPCT. Reglamento de Trabajos Fin de Estudios (Grado y Máster) en la Universidad Politécnica de Cartagena. Aprobado en Consejo de Gobierno del 7 de febrero de 2020.

ETSAE. Reglamento de Trabajos Fin de Estudios en la ETSAE. Universidad Politécnica de Cartagena. Aprobado en Consejo de Gobierno del 26 de febrero de 2020.

PÉREZ SÁNCHEZ, J.C. et al. (2019). Análisis, discusión y propuestas de mejora en el desarrollo de la asignatura Proyecto Fin de Grado del Grado en Arquitectura Técnica. En Memoria del Programa de Redes-I3CE de calidad, innovación e investigación en docencia univeritaria. Convocatoria 2018-19. Universidad de Alicante; pp. 2749-2766.

RAPOSO RIVAS, M.; ZABALZA BERAZA, M.A. (2011) La formación práctica de estudiantes: repensando el Practicum. Revista de Educación, n³54. Madrid. Ministerio de Educación; pp. 17-20. 
SÁNCHEZ CARRACEDO et al. (2015). El método socrático como guía del Trabajo Fin de Grado. En ReVisión, vol 8, n¹. UPC. Barcelona; pp. 53-62.

SANCHO SÁEZ, J. (2019). Técnicas de enseñanza para mejorar la motivación de los estudiantes. Revista Virtual Educrea. Biblioteca Docente.

VALVERDE CANTERO, D.; ARTEAGA MARTínEZ, J.J. y ALFARO GONZÁLEZ, J. (2012). Coordinación multidiscipplinar de PFGS en la titulación de Grado en Ingeniería de Edificación. En XVI Congreso Internacional de Ingeniería de Proyectos. Valencia, AEIPRO. Universidad Politécnica de Valencia; pp. 2511-2519.

ZABALZA BERAZA, M.A. (2011). El prácticum en la formación universitaria: estado de la cuestión. Revista de Educación n³54. Madrid. Ministerio de Educación; pp. 21-43. 